\title{
Inequalities for Phase-space Rényi entropies
}

\author{
I. Hornyák and Á. Nagy \\ Department of Theoretical Physics, University of Debrecen, \\ H-4010 Debrecen, Hungary
}

8th March 2011

\begin{abstract}
Relations between different kinds of phase-space Rényi and Shannon entropies are proved.
\end{abstract}

\section{Introduction}

There has been a growing interest in information theoretical concepts in many branches of physics. Rényi [2] introduced a one-parameter extension of Shannon information [1] as early as 1961, still this kind of information has only recently obtained a wide range of application ( e. g. $[3,4,5,6]$ ). Rényi entropy of atoms were studied in both position and momentum spaces [7]. Motivated by these studies now we introduce phase-space Rényi entropy based on a special family of phase-space distribution functions. Phase-space representation of quantum mechanics has received attention in several fields of physics (e.g. [8, 9, 10, 11, 12, 13, 14, 15, 16]).

In this paper we present theorems for phase-space Rényi entropy. Earlier phase-space Rényi entropy was obtained from the Wigner and the Husimi distribution functions. Here another family of distribution functions are studied.

The paper is organized as follows: In section II we give phase-space distribution functions studied in this paper and the Rényi entropy and summarize the corresponding uncertainty relation. Section III presents theorems for the Rényi and Shannon entropy and their proofs. The last section is devoted to discussion. 


\section{Phase-space distribution functions and phase- space Rényi entropy}

We consider a distribution function $F(q, p)$ with the properties

$$
\begin{gathered}
\int F(q, p) \mathrm{d} p=|\psi(q)|^{2}=\varrho(q), \\
\int F(q, p) \mathrm{d} q=|\phi(p)|^{2}=\gamma(p)
\end{gathered}
$$

and

$$
F \geq 0
$$

$\psi(q)$ and $\phi(p)$ are the configuration and momentum wave functions, respectively. $\varrho(q)$ and $\gamma(p)$ are the configuration-space and momentum-space densities, respectively. Both $\varrho(q)$ and $\gamma(p)$ and consequently $F$ are normalized to 1 .

$$
\int F \mathrm{~d} q \mathrm{~d} p=1
$$

The distribution function $F(q, p)$ can conveniently be written as

$$
F(q, p)=\rho(q) \gamma(p) f(q, p)
$$

From relations (1) - (5) follows that $f \geq 0$. We study the special case where the degree of freedom is 1 . The extension to any general case is straightforward.

We mention in passing that these distribution function $F(q, p)$ are not of Wigner-type [8]. The Wigner function cannot be considered a proper probability distribution because it may take on negative values. Since Wigner's original paper several other distrubution functions have been found which satisfy the conditions (1) and (2). Wigner proved [9] that positive distributions which bilinear in the wave function do not exist. Later Cohen and Zaparovanny [17] proved that positive distributions satisfying the conditions (1) and (2) exist, though these are not bilinear in the wave function. 
Consider $\zeta$-order associated escort distribution functions $\rho_{\zeta}(q)$

$$
\rho_{\zeta}(q)=\frac{\rho(q)^{\zeta}}{\int \mathrm{d} q \rho(q)^{\zeta}}
$$

in position space and $\zeta$-order associated escort distribution functions $\gamma_{\zeta}(p)$

$$
\gamma_{\zeta}(p)=\frac{\gamma(p)^{\zeta}}{\int \mathrm{d} p \gamma(p)^{\zeta}}
$$

in momentum space with real $\zeta$. In this paper the case $\zeta=2$ is studied. We can construct a phase-space distribution function $\tilde{F}(q, p)$ from the 2 nd-order escort marginal distribution functions $\rho_{2}(q)$ and $\gamma_{2}(p)$ with the definition

$$
\tilde{F}(q, p)=\rho_{2}(q) \gamma_{2}(p)
$$

We can also write

$$
\tilde{F}(q, p)=\frac{\rho^{2} \gamma^{2}}{\langle\rho\rangle\langle\gamma\rangle}
$$

where

$$
\langle\rho\rangle=\int \rho^{2} \mathrm{~d} q
$$

and

$$
\langle\gamma\rangle=\int \gamma^{2} \mathrm{~d} p
$$

The distribution function $\tilde{F}(q, p)$ satisfies conditions (3) and (4). The marginal densities of $\tilde{F}(q, p)$, however, are different from the ones of $F(q, p)$ :

$$
\int \tilde{F} \mathrm{~d} q=\gamma_{r} \gamma, \quad \gamma_{r}=\frac{\gamma}{\langle\gamma\rangle}
$$

and

$$
\int \tilde{F} \mathrm{~d} p=\rho_{r} \rho, \quad \rho_{r}=\frac{\rho}{\langle\rho\rangle}
$$

Rényi entropy of order $\alpha$ for a $D$ dimensional probability density function $g\left(r_{1}, \ldots, r_{D}\right)$ normalized to one is defined by

$$
R_{g}^{\alpha} \equiv \frac{1}{1-\alpha} \ln \int g^{\alpha}(\mathbf{r}) \mathrm{d} \mathbf{r} \quad \text { for } \quad 0<\alpha<\infty \quad \alpha \neq 1,
$$


where $\mathbf{r}$ stands for $r_{1}, \ldots, r_{D}$. The Rényi entropy tends to the Shannon entropy when $\alpha \rightarrow 1$ :

$$
S_{g}=-\int g(\mathbf{r}) \ln g(\mathbf{r}) \mathrm{d} \mathbf{r} .
$$

Recently, several entropic uncertainty relations were proposed. These give lower bounds to the sum of the Shannon information entropies [18, 19, 20, 21]. The first relations were obtained in [18] for the sum of position and momentum space Shannon entropies. The proposal to use one-electron densities in the position and the momentum spaces was first given by Gadre [19].

Bialynicki-Birula derived an uncertainty relation [22] for the Rényi entropy sum:

$$
\begin{gathered}
R_{\rho}^{\alpha}+R_{\gamma}^{\beta} \geq h(\alpha, \beta), \quad \frac{1}{\alpha}+\frac{1}{\beta}=2 \\
h(\alpha, \beta)=\frac{3 N}{2}\left[\frac{1}{\alpha-1} \ln \left(\frac{\alpha}{\pi}\right)+\frac{1}{\beta-1} \ln \left(\frac{\beta}{\pi}\right)\right]
\end{gathered}
$$

This uncertainty relation reaches the Shannon entropic uncertainty relation [18]

$$
S_{\rho}+S_{\gamma} \geq 3(1+\ln \pi)
$$

in the limit $\alpha \rightarrow 1$. Entropic uncertainty relations were also derived for finite-dimensional Hilbert spaces [23, 24, 25, 26, 27, 28, 29, 30, 31]. For a recent summary see [32]. Recently, the maximum Rényi entropy principle has been applied to generalize the Thomas-Fermi model [33].

Now the phase-space Rényi entropy of order $\alpha$ is given by

$$
R_{F}^{\alpha} \equiv \frac{1}{1-\alpha} \ln \int F^{\alpha}(\mathbf{r}) \mathrm{d} \mathbf{r d} \mathbf{p} \quad \text { for } \quad 0<\alpha<\infty \quad \alpha \neq 1 .
$$

We will also consider the Rényi entropies $R_{f}^{\alpha}, R_{\rho}^{\alpha}$ and $R_{\gamma}^{\alpha}$.

\section{Theorems for Rényi entropy}

Consider the distribution functions of configuration space (Eq. (1)), momentum space (Eq. (2)) and phase-space (Eq. (5)). Suppose that the Rényi entropies $R_{f}^{\alpha}, R_{\rho}^{\alpha}$ and $R_{\gamma}^{\alpha}$ exist, where $f$ is given by Eq. (5). Then the following inequality holds between them: 


\section{Theorem 1}

$$
R_{f}^{\alpha} \leq(1-\beta)\left[R_{\rho}^{\beta}+R_{\gamma}^{\beta}\right], \quad \alpha^{-1}+\beta^{-1}=1, \quad \alpha, \beta>1
$$

\section{Proof:}

Substituting first Eq. (5) into (4) and making use of Hölder's inequality [34] we can write

$$
\begin{aligned}
\int F \mathrm{~d} q \mathrm{~d} p & =\int \mathrm{d} p \gamma \int \mathrm{d} q \rho f \\
& \leq \int \mathrm{d} p \gamma\left[\left(\int \rho^{x} \mathrm{~d} q\right)^{\frac{1}{x}}\left(\int f^{y} \mathrm{~d} q\right)^{\frac{1}{y}}\right] \\
& =\left(\int \rho^{x} \mathrm{~d} q\right)^{\frac{1}{x}} \int \mathrm{d} p \gamma\left(\int f^{y} \mathrm{~d} q\right)^{\frac{1}{y}} \\
& \leq\left(\int \rho^{x} \mathrm{~d} q\right)^{\frac{1}{x}}\left(\int \gamma^{\tilde{x}} \mathrm{~d} p\right)^{\frac{1}{\tilde{x}}}\left(\int\left[\int f^{y} \mathrm{~d} q\right]^{\frac{\tilde{y}}{y}} \mathrm{~d} p\right)^{\frac{1}{\tilde{y}}}
\end{aligned}
$$

where

$$
x^{-1}+y^{-1}=1 \quad \tilde{x}^{-1}+\tilde{y}^{-1}=1
$$

and

$$
x, y, \tilde{x}, \tilde{y}>1 .
$$

Taking into account that the logarithm function is strictly monotonic, applying Eq. (4) and taking $x=\tilde{x}=\beta$ and $y=\tilde{y}=\alpha$ inequality (21) has the form

$$
\begin{aligned}
& 0 \leq \frac{1-\beta}{\beta} R_{\rho}^{\beta}+\frac{1-\beta}{\beta} R_{\gamma}^{\beta}+\frac{\beta-1}{\beta}\left(1-\frac{\beta}{\beta-1}\right) R_{f}^{\alpha} \\
& 0 \leq \frac{1}{\beta}\left[(1-\beta) R_{\rho}^{\beta}+(1-\beta) R_{\gamma}^{\beta}-R_{f}^{\alpha}\right]
\end{aligned}
$$

with $\beta>1>0$. From inequality (24) follows theorem 1 .

The Rényi entropies $R_{f}^{\alpha}, R_{\rho}^{\alpha}$ and $R_{\gamma}^{\alpha}$ in Theorem 1 can be related to the phase-space Rényi information entropy $R_{F}^{\frac{\alpha}{2}}$ :

\section{Theorem 2}

Suppose that the Rényi entropies $R_{f}^{\alpha}, R_{\rho}^{\alpha}, R_{\gamma}^{\alpha}$ and $R_{F}^{\frac{\alpha}{2}}$ exist, where $f$ is 
given by Eq. (5). Then the following inequality holds:

$$
\begin{array}{rrr}
R_{F}^{\frac{\alpha}{2}} \leq \frac{1-\alpha}{2-\alpha}\left[R_{\rho}^{\alpha}+R_{\gamma}^{\alpha}+R_{f}^{\alpha}\right] & , \text { if } & 0<\alpha<2 \\
R_{F}^{\frac{\alpha}{2}} \geq \frac{1-\alpha}{2-\alpha}\left[R_{\rho}^{\alpha}+R_{\gamma}^{\alpha}+R_{f}^{\alpha}\right], & \text { if } & 2<\alpha
\end{array}
$$

Proof:

$$
\begin{aligned}
\int F^{\alpha} \mathrm{d} q \mathrm{~d} p & =\int \mathrm{d} p \gamma^{\alpha} \int \mathrm{d} q \rho^{\alpha} f^{\alpha} \\
& \leq \int \mathrm{d} p \gamma^{\alpha}\left[\left(\int \rho^{2 \alpha} \mathrm{d} q\right)^{\frac{1}{2}}\left(\int f^{2 \alpha} \mathrm{d} q\right)^{\frac{1}{2}}\right] \\
& =\left(\int \rho^{2 \alpha} \mathrm{d} q\right)^{\frac{1}{2}} \int \mathrm{d} p \gamma^{\alpha}\left(\int f^{2 \alpha} \mathrm{d} q\right)^{\frac{1}{2}} \\
& \leq\left(\int \rho^{2 \alpha} \mathrm{d} q\right)^{\frac{1}{2}}\left(\int \gamma^{2 \alpha} \mathrm{d} p\right)^{\frac{1}{2}}\left(\int f^{2 \alpha} \mathrm{d} q \mathrm{~d} p\right)^{\frac{1}{2}}
\end{aligned}
$$

where Schwarz's inequality was used. Taking into account again that the logarithm function is strictly monotonic the inequality (26) takes the form

$$
(1-\alpha) R_{F}^{\alpha} \leq\left(\frac{1}{2}-\alpha\right)\left[R_{\rho}^{2 \alpha}+R_{\gamma}^{2 \alpha}+R_{f}^{2 \alpha}\right], \quad \alpha>0 .
$$

After replacing $\alpha$ by $\frac{\alpha}{2}$ inequality (27) leads to

$$
(2-\alpha) R_{F}^{\frac{\alpha}{2}} \leq(1-\alpha)\left[R_{\rho}^{\alpha}+R_{\gamma}^{\alpha}+R_{f}^{\alpha}\right], \quad \alpha>0 .
$$

From inequality (28) immediatelly follows theorem 2.

We can establish a relation between the phase-space Rényi entropy $R_{F}^{\frac{\alpha}{2}}$ and the Rényi entropies constructed from the position- and momentum-space marginal densities:

\section{Theorem 3}

Suppose that the Rényi entropies $R_{\rho}^{\alpha}, R_{\gamma}^{\alpha}$ and $R_{F}^{\frac{\alpha}{2}}$ exist. Then the following inequality holds:

$$
R_{F}^{\frac{\alpha}{2}} \leq \frac{1}{2-\beta}\left[R_{\rho}^{\alpha}+R_{\gamma}^{\alpha}\right]+\frac{1}{2-\alpha}\left[R_{\rho}^{\beta}+R_{\gamma}^{\beta}\right], \quad 1<\alpha<2, \quad \alpha^{-1}+\beta^{-1}=1 .
$$




\section{Proof:}

Substituting inequality (20) into inequality (25) and making use of the equalities

$$
1-\beta=\frac{1}{1-\alpha}
$$

and

$$
\frac{1-\alpha}{2-\alpha}=\frac{1}{2-\beta}
$$

theorem 3 follows.

Now, we apply distribution function $\tilde{F}(q, p)$ constructed from 2nd-order escort marginal distribution functions to derive equalities. The Shannon entropy corresponding to the distribution function $\tilde{F}(q, p)$ is given by

$$
\tilde{S}=-\int \tilde{F} \ln \tilde{F}
$$

Introducing the notations $g=\rho \gamma$ and $\eta=\int g^{2} \mathrm{~d} q \mathrm{~d} p=\langle\rho\rangle\langle\gamma\rangle$ the Shannon entropy $\tilde{S}$ can be written as

$$
\tilde{S}=-\frac{1}{\eta} \int g^{2} \ln g^{2}+\ln \eta .
$$

Then holds

\section{Theorem 4}

$$
\tilde{S}=2 \lim _{\alpha \rightarrow 2} \frac{\mathrm{d} R_{g}^{\alpha}}{\mathrm{d} \alpha}-\ln \eta
$$

Proof:

From the definition of the Rényi entropy follows that

$$
\begin{aligned}
\frac{\mathrm{d} R_{g}^{\alpha}}{\mathrm{d} \alpha} & =-\frac{1}{(1-\alpha)^{2}}(-1) \ln \int g^{\alpha}+\frac{1}{1-\alpha} \frac{1}{\int g^{\alpha}} \int g^{\alpha} \ln g \\
& =\frac{1}{(1-\alpha)^{2}} \ln \int g^{\alpha}+\frac{1}{1-\alpha} \frac{\int g^{\alpha} \ln g}{\int g^{\alpha}} .
\end{aligned}
$$

Taking the limit $\alpha \rightarrow 2$ we obtain

$$
\lim _{\alpha \rightarrow 2} \frac{\mathrm{d} R_{g}^{\alpha}}{\mathrm{d} \alpha}=\ln \int g^{2}-\frac{\int g^{2} \ln g}{\int g^{2}} .
$$

From Eqs. (33) and (36) we arrive at theorem 4. 
The phase-space Shannon entropy $\tilde{S}$ can be expressed in a symmetric form according to the following theorem.

\section{Theorem 5}

$$
\tilde{S}=\lim _{\alpha \rightarrow 2}\left(\frac{1}{2-\beta} R_{g}^{\alpha}+\frac{1}{2-\alpha} R_{g}^{\beta}\right)
$$

\section{Proof:}

Notice first that $\frac{g^{\beta}}{g^{\alpha}} \rightarrow 1$ as $\alpha \rightarrow 2$. Then making use of Eq.(31) we obtain

$$
\frac{1}{2-\beta} R_{g}^{\alpha}+\frac{1}{2-\alpha} R_{g}^{\beta}=R_{g}^{\alpha}+\frac{1}{2-\alpha}\left(R_{g}^{\beta}-R_{g}^{\alpha}\right) .
$$

The difference in the second term can be written as

$$
R_{g}^{\beta}-R_{g}^{\alpha}=\ln \left(\frac{\int g^{\beta}}{\int g^{\alpha}}\right)+\ln \left(\frac{\left(\int g^{\alpha}\right)^{\beta}}{\left(\int g^{\beta}\right)^{\alpha}}\right) .
$$

The limit $\alpha \rightarrow 2$ in Eq.(37) can be easily calculated by L'Hospital's rule:

$$
\lim _{\alpha \rightarrow 2} \frac{R_{g}^{\beta}-R_{g}^{\alpha}}{2-\alpha}=-\frac{1}{\int g^{2}} \int g^{2} \ln g^{2}+2 \ln \int g^{2}
$$

Taking into account that

$$
R_{g}^{\alpha} \underset{\alpha \rightarrow 2}{\longrightarrow}-\ln \int g^{2}
$$

Eq.(40) leads to Eq.(37).

There exists an inequality between $S_{F}$ and $\tilde{S}$.

\section{Theorem 6}

$$
S_{F} \leq \tilde{S}
$$

\section{Proof:}

Taking into account that $S_{g}=S_{\rho}+S_{\gamma}$ theorem 6 immediatelly follows from theorems 3 and 5 .

As a consequence of theorem 6 we are led to the inequality:

$$
S_{F} \leq \lim _{\alpha \rightarrow 2}\left(\frac{1}{2-\beta} R_{g}^{\alpha}+\frac{1}{2-\alpha} R_{g}^{\beta}\right)
$$




\section{Discussion}

As an illustration consider atomic ions with a single electron and atomic number $Z$. The corresponding Rényi entropies have recently been calculated [35]:

$$
\begin{aligned}
& R_{\rho}^{\alpha, \text { H-like }}=\frac{3 \ln \alpha}{\alpha-1}-\ln \frac{Z^{3}}{\pi} \\
& R_{\gamma}^{\alpha, \text { H-like }}=\frac{1}{1-\alpha} \ln \left[\frac{8^{\alpha+1} \pi^{5 / 2-3 \alpha} \Gamma(4 \alpha+1 / 2) \Gamma(8 \alpha-3)}{\Gamma(8 \alpha) \Gamma(4 \alpha-1)}\right]+\ln \frac{Z^{3}}{\pi}
\end{aligned}
$$

Substituting Eqs. (44) and (45) into inequality (20) we obtain

$$
R_{f}^{\alpha, \mathrm{H}-\mathrm{like}} \leq \ln G(\beta), \quad \alpha^{-1}+\beta^{-1}=1, \quad \alpha, \beta>1,
$$

where

$$
G(\beta)=\frac{\beta^{-3} 8^{\beta+1} \pi^{5 / 2-3 \beta} \Gamma(4 \beta+1 / 2) \Gamma(8 \beta-3)}{\Gamma(8 \beta) \Gamma(4 \beta-1)}
$$

for any $f$ satisfying conditions (1) and (5).

Substituting Eqs. (44) and (45) into inequality (29) we are led to

$$
R_{F}^{\frac{\alpha}{2}, \text { H-like }} \leq \frac{1}{2-\alpha} \ln G(\alpha)+\frac{1}{2-\beta} \ln G(\beta) \quad, \quad 1<\alpha<2, \quad \alpha^{-1}+\beta^{-1}=1 .
$$

Taking the limit $\alpha \rightarrow 1$ we arrive at the Shannon entropy sum

$$
S_{\rho}^{\mathrm{H}-\mathrm{like}}+S_{\gamma}^{\mathrm{H}-\mathrm{like}}=\ln \left(2^{5} \pi^{3}\right)-\frac{1}{3} \approx 6.567
$$

We can also calculate $\tilde{S}$

$$
\tilde{S}^{\mathrm{H}-\mathrm{like}}=\ln \left(2^{3} \pi^{3} 33\right)-\frac{21859}{3465} \approx 2.70163
$$

Substituting $\tilde{S}$ into inequality (42) we obtain

$$
S_{\mathrm{F}}^{\mathrm{H}-\mathrm{like}} \leq 2.70163
$$

Note that the upper bounds universal in the sense that there is no dependence on the atomic number.

In this paper relations between different kinds of phase-space Rényi and Shannon entropies are established. In deriving these equalities and inequalities the position and momentum space marginal densities have a crucial role. 
In the uncertainty relation (16) there are Rényi entropies with different orders $\alpha$ and $\beta$. Theorems 1 and 2 provide inequalities containing Rényi entropies arising from the position and momentum space marginal densities with the same order.

Writing the phase-space distribution function $F$ as a product of the functions $\rho, \gamma$ and $f$ (Eq. (5)) theorem 2 gives an inequality for the phase-space Rényi entropy expressed as a sum of entropies constructed from the functions $\rho, \gamma$ and $f$. It is very interesting that the order of the phase-space Rényi entropy is half of the order of the composite Rényi entropies. Note that the inequality sign changes around the Shannon case. For the Shannon case there is a separate inequality formalized in theorem 6 . It gives an upper bound for the phase-space Shannon entropy $S_{F}$. It is remarkable that the upper bound is another phase-space Shannon information entropy $\tilde{S}_{F}$ constructed from the second order escort marginal distribution functions that does not depend on $f$. That is, for a given wave function the upper bound is fixed, while $S_{F}$ changes if we change $f$.

The expectation values of the second-order escort marginal densities (10) and (11) are well known quantities. They represent the so called disequilibrium or the distance from equilibrium (most probable state). They are fundamental ingredients of the continuous version of the LMC (López-RuizMancini-Calbet) complexity measure is [36].

\section{References}

[1] C. E. Shannon, Bell Syst. Tech. J. 27, 379 (1948).

[2] A. Rényi, in Proceedings of Fourth Berkeley Symp. on Mathematics, Statistics and Probability, vol.1. Univ. California Press, Berkeley, 1961, pp. 547 .

[3] O. Gühne and M. Lewenstein, Phys. Rev. A 70, 022316 (2004); G. Adesso, A. Serafini and F. Illuminati, Phys. Rev. A 70, 022318 (2004); A. Bovino, G. Castagnolli, A. Ekert, P. Horodecki, C. M. Alves and A. V. Serfienko, Phys. Rev. Lett. 95, 240407 (2005). 
[4] R. Renner, N. Gisin and B. Kraus, Phys. Rev. A 72, 012332 (2005); V. Giovanetti and S. Lloyd, Phys. Rev. A 69, 062307 (2004).

[5] P. Lévay, S. Nagy and J. Pipek Phys. Rev. A 72, 022302 (2005).

[6] D. G. Arbo, C. O. Reinhold, J. Burgdörfer, A. K. Pattanayak, C. L. Stokely, W. Zhao, J. C. Lancaster and F. B. Dunning, Phys. Rev. A 67, 063401 (2003).

[7] E. Romera and Á. Nagy, Phys. Lett. A 3724918 (2008).

[8] E. Wigner, Phys. Rev. 40, 749 (1932).

[9] E. Wigner, Quantum-Mechanical Distribution Functions Revisited, in Perspectives in Quantum Theory, ed. by W. Yourgrau and A. van der Merwe (M. I. T., Cambridge, 1971).

[10] J. P. Dahl, Theor. Chim. Acta, 81, 329 (1982); M. Springborg and J. P. Dahl, in Local Density Approximations in Quantum Chemistry and Solid State Physics, ed. by J. P. Dahl and J. Avery ( Plenum, 1984); M. Springborg and J. P. Dahl, Phys. Rev. A 36, 1050 (1997); J. P. Dahl and M. Springborg, J. Chem. Phys. 88, 4535 (1988); J. P. Dahl and W. P. Schleich, Phys. Rev. A 65, 022109 (2002).

[11] K. Husimi, Proc. Phys. Math. Soc. Japan 22, 264 (1940); M. E. Casida, J. E. Harriman and J. L. Anchell, Int. J. Quantum Chem. Symp. 21, 435 (1987); J. E. Harriman, J. Chem. Phys. 88, 6399 (1988); J. L. Anchell and J. E. Harriman, J. Chem. Phys. 89, 6860 (1988).

[12] J. J. Wlodarz, J. Chem. Phys. 100, 7476 (1994); Int. J. Quantum Chem. 51, 123 (1994); Int. J. Quantum Chem. 56, 233 (1995).

[13] Q. S. Li, G. M. Wei and L. Q. Lu, Phys. Rev. A 70, 022105 (2004).

[14] J. Dunkel and S. A. Trigger, Phys. Rev. A 71, 052102 (2005).

[15] I. Varga and J. Pipek, Phys. Rev. E 68, 026202 (2003).

[16] R. Leipnik, Inf. Control. 2, 64 (1959). 
[17] L. Cohen and Y. I. Zaparovanny, J. Math. Phys. 21, 794 (1980).

[18] I. Bialynicki-Birula and J. Mycielski, Commun. Math. Phys. 44, 129 (1975).

[19] S. R. Gadre, Phys. Rev. A 30, 620 (1984).

[20] S. R. Gadre, S. B. Sears, S. J. Chakravorty and R. D. Bendale, Phys. Rev. A 32, 2602 (1985).

[21] S. R. Gadre and R. D. Bendale, Int. J. Quantum Chem. 28, 311 (1985).

[22] I. Bialynicki-Birula, Phys. Rev. A 74, 052101 (2006).

[23] D. Deutsch, Phys. Rev. Lett. 50, 631 (1983).

[24] M. H. Partovi, Phys. Rev. Lett. 50, 1883 (1983).

[25] J. B. M. Uffink and J. Hilgewoord, Found. Phys. 15, 925 (1985).

[26] K. Kraus, Phys. Rev. D 35, 3070 (1987).

[27] H. Massen and J. B. M. Uffink, Phys. Rev. Lett. 60, 1103 (1988).

[28] J. Sánches, Phys. Lett. A 173, 233 (1993).

[29] M. J. W. Hall, Phys. Rev. Lett. 74, 3307 (1995).

[30] J. Sánches-Ruiz, Phys. Lett. A 244, 189 (1998).

[31] J. I. de Vicente and J. Sánches-Ruiz, Phys. Rev. A 77, 042110 (2008).

[32] Á. Nagy, Acta Physica Debrecina 4337 (2009).

[33] Á. Nagy and E. Romera, Phys. Lett. A 373844 (2009).

[34] G.H. Hardy, J.E. Littlewood, G. Pólya, Inequalities, Cambridge University Press, 1934

[35] E. Romera and Á. Nagy, Phys. Lett. A 372, 4918 (2008).

[36] R. G. Catalan, J. Garay, and R. Lopez-Ruiz, Phys. Rev. E 66011102 (2002). 


\section{Acknowledgements}

This work is supported by TAMOP 4.2.1-08/1-2008-003 project. The project is implemented through the New Hungary Development Plan co-financed by the European Social Fund, and the European Regional Development Fund. Grant OTKA No. K 67923 is also gratefully acknowledged. 